\title{
GALERKIN APPROXIMATION AND THE STRONG SOLUTION OF THE NAVIER-STOKES EQUATION
}

\author{
HANNELORE BRECKNER \\ Martin-Luther Universität Halle-Wittenberg \\ Fachbereich Mathematik und Informatik \\ Institut für Optimierung und Stochastik \\ D-06099 Halle (Saale), Germany
}

(Received June, 1998; Revised June, 1999)

\begin{abstract}
We consider a stochastic equation of Navier-Stokes type containing a noise part given by a stochastic integral with respect to a Wiener process. The purpose of this paper is to approximate the solution of this nonlinear equation by the Galerkin method. We prove the convergence in mean square.

Key words: Stochastic Navier-Stokes Equation, Itô Integral, Galerkin Method.
\end{abstract}

AMS subject classifications: $60 \mathrm{H} 15,60 \mathrm{H} 30$.

\section{Introduction}

The Navier-Stokes equation has important physical and technical applications. It describes the behavior of a viscous velocity field of an incompressible liquid.

Important results concerning the theory and numerical analysis of the deterministic Navier-Stokes equation can be found in the book of R. Temam [16]. The author also presents in this book, the Galerkin method for this equation, which is one of the well-known methods in the theory of partial differential equations that is used to prove existence properties and to obtain finite dimensional approximations for the solutions of the equations. The deterministic Galerkin method was adapted to the stochastic case, for example, in the papers of W. Grecksch, P.E. Kloeden [5], I. Gyöngy [8], and E. Pardoux [13], where the authors investigate evolution equations with Lipschitz continuous nonlinearities. But the method can also be used for the stochastic Navier-Stokes equation, which does not have Lipschitz continuous nonlinearities. The Galerkin method for the stochastic Navier-Stokes equation has been investigated for example from A. Bensoussan [1], M. Capinski, N.J. Cutland [2], D. Gatarek [3], A.I. Komech, M.I. Vishik [10], B. Schmalfuß [14], and M. Voit [17].

The stochastic Navier-Stokes equation on the domain of flow $G \subset \mathbb{R}^{n}(n \geq 2)$ is given by 


$$
\begin{gathered}
\frac{\partial u}{\partial t}-\nu \Delta u=-(u, \nabla) u+f-\nabla p+\odot u \frac{\partial w}{\partial t} \\
\operatorname{div} u=0, \quad u(0, x)=u_{0}(x),\left.u(t, x)\right|_{\partial G}=0, \quad t>0, \quad x \in G,
\end{gathered}
$$

where $u$ is the velocity field, $\nu$ is the viscosity, $\Delta$ is the Laplacian, $\nabla$ is the gradient, $f$ is an external force, $p$ is the pressure, $u_{0}$ is the initial condition. The term $e u \frac{\partial w}{\partial t}$ describes a state dependent random noise. This equation can be written equivalently in the form of an evolution equation, using certain vector spaces and operators.

In the study of evolution equations of Navier-Stokes type one can consider weak solutions of martingale type or strong solutions. The above-mentioned papers consider weak (statistical) solutions. The techniques used in the proofs are the construction of the Galerkin-type approximations of the solutions and some a priori estimates, that allow one to prove compactness properties of the corresponding probability measures and finally to obtain a solution of the equation (using Prokhorov's criterion and Skorokhod's theorem).

In this paper we consider strong solutions of the Navier-Stokes equation, i.e., we define the Navier-Stokes equation in the generalized sense as an evolution equation, assuming that the stochastic processes are defined on a given complete probability space and the Wiener process is given in advance. The aim of this paper is to prove the existence of the strong solution of the Navier-Stokes equation by approximating it by means of the Galerkin method, i.e., by a sequence of solutions of finite dimensional evolution equations (see equations $\left(P_{n}\right)$ ). Since we consider the strong solution of the Navier-Stokes equation, we do not need to use the techniques considered in the case of weak solutions. The techniques applied in this paper use in particular, the properties of stopping times and some basic convergence principles from functional analysis. An important result, which cannot be proved in the case of weak solutions, is that the Galerkin-type approximations converge in mean square to the solution of the Navier-Stokes equation (see Theorem 2.3).

The structure of the paper is as follows: in Section 2 we formulate the main results of the paper (Theorem 2.2 and Theorem 2.3) and in Section 3 we give the proofs for these results, while Section 4 contains some convergence principles from functional analysis that we use in our proofs.

\section{Formulation of the Problem}

First we state the assumptions about the stochastic evolution equation to be considered.

(i) $\left(V, H, V^{*}\right)$ is an evolution triple (see $\left[19\right.$, p. 416]), where $\left(V,\|\cdot\|_{V}\right)$ and $(H,\|\cdot\|)$ are separable Hilbert spaces, $V^{*}$ is the dual space of $V$, and the embedding operator $V \subset H$ is assumed to be compact. $\left\langle v^{*}, v\right\rangle$ denotes the application of $v^{*} \in V^{*}$ to $v \in V$, while $(\cdot, \cdot)$ denotes the scalar product in $H$.

(ii) $\mathcal{A}: V \rightarrow V^{*}$ is a linear operator such that $\langle\mathcal{A} v, v\rangle \geq \nu\|v\|_{V}^{2}$ for all $v \in V$ $(\nu>0$ is a constant) and $\langle\mathcal{A} u, v\rangle=\langle\mathcal{A} v, u\rangle$ for all $u, v \in V$.

(iii) $\mathscr{B}: V \times V \rightarrow V^{*}$ is a bilinear operator such that

$$
\langle\mathfrak{B}(u, v), v\rangle=0 \text { for all } u, v \in V
$$


and for which there exists a positive constant $b>0$ such that

$$
|\langle\mathscr{B}(u, v), z\rangle|^{2} \leq b\|z\|_{V}^{2}\|u\|\|u\|_{V}\|v\|\|v\|_{V} .
$$

(iv) $\quad \mathrm{C}: V \rightarrow H$ is a mapping with $\mathrm{C} 0=0$ and

$$
\|C u-C v\|^{2} \leq \lambda\|u-v\|^{2}
$$

for all $u, v \in V$, where $\lambda$ is a positive constant.

(v) $(\Omega, \mathcal{F}, P)$ is a complete probability space and $\left(\mathcal{F}_{t}\right)_{t \in[0, T]}$ a right-continuous

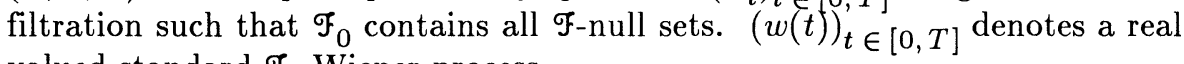
valued standard $\mathcal{F}_{t}$-Wiener process.

(vi) $f$ is a progressively measurable $H$-valued process for which $E \int_{0}^{T}\|f(s)\|^{4} d s<\infty$.

(vii) $x_{0}$ is a $H$-valued $\mathscr{F}_{0}$ measurable random process such that $E\left\|x_{0}\right\|^{4}<\infty$.

An adapted $V$-valued process $(u(t))_{t \in[0, T]}$ with $E\|u(t)\|^{2}<\infty$ for all $t \in[0, T]$ and $E \int_{0}^{T}\|u(t)\|_{V}^{2} d t<\infty$ is called a strong solution of the stochastic Navier-Stokes equation if it satisfies the equation:

$$
\begin{aligned}
(u(t), v)+ & \int_{0}^{t}\langle\mathcal{A} u(s), v\rangle d s=\left(x_{0}, v\right)+\int_{0}^{t}\langle\mathscr{B}(u(s), u(s)), v\rangle d s \\
& +\int_{0}^{t}(f(s), v) d s+\int_{0}^{t}(\mathcal{C} u(s), v) d w(s)
\end{aligned}
$$

for all $v \in V, t \in[0, T]$ and for a.e. $\omega \in \Omega$, where the stochastic integral is understood in the Itô sense. As usual, in the notation of random variables or stochastic processes we generally omit the dependence on $\omega$.

Notation: Let $\left(S, \mid \cdot \|_{S}\right)$ be a Banach space and denote by $\mathcal{L}_{S}^{2}(\Omega)$ the linear space of all functions $u: \Omega \rightarrow S$ that are $\mathscr{F}$-measurable and $E\|u\|_{S}^{2}<\infty$. Further, denote by $\ell_{S}^{2}(\Omega \times[0, T])$ the linear space of all processes $u: \Omega \times[0, T] \rightarrow S$ that are $\mathscr{F} \times \mathscr{B}_{[0, T]^{-}}$measurable, adapted to the filtration $\left(\mathscr{F}_{t}\right)_{t \in[0, T]}$ and $E \int_{0}^{T}\|u(t)\|_{S}^{2} d t<\infty$. Weak convergence is denoted by $\rightarrow$.

Let $h_{1}, h_{2}, \ldots, h_{n}, \ldots \in H$ be the eigenvectors of the operator $\mathcal{A}$, for which we consider the domain of definition $\operatorname{Dom}(\mathcal{A})=\{v \in V \mid \mathcal{A} v \in H\}$. These eigenvectors form an orthonormal base in $H$ (see [12, p. 110]). For each natural number $n$ we consider $H_{n}=\operatorname{sp}\left\{h_{1}, h_{2}, \ldots, h_{n}\right\}$ equipped with the norm induced from $H$. Let $\mathcal{A}_{n}: H_{n} \rightarrow H_{n}, \mathscr{B}_{n}: H_{n} \times H_{n} \rightarrow H_{n}, \mathrm{C}_{n}: H_{n} \rightarrow H_{n}$ be defined respectively by

$$
\mathcal{A}_{n} u=\sum_{i=1}^{n}\left\langle\mathcal{A} u, h_{i}\right\rangle h_{i}, \mathscr{B}_{n}(u, v)=\sum_{i=1}^{n}\left\langle\mathscr{B}(u, v), h_{i}\right\rangle h_{i}, \quad \mathrm{C}_{n} u=\sum_{i=1}^{n}\left(\mathcal{C} u, h_{i}\right) h_{i}
$$

for all $u, v \in H_{n}$. For every $f \in \mathcal{L}_{H}^{2}(\Omega \times[0, T])$, we consider the partial sums

$$
f_{n}(t)=\sum_{i=1}^{n}\left(f(t), h_{i}\right) h_{i}
$$

for all $t \in[0, T]$ and a.e. $\omega \in \Omega$.

For each natural number $n=1,2,3, \ldots$, we consider the sequence of finite dimen- 
sional evolution equations

$$
\begin{aligned}
\left(u_{n}(t), v\right) & +\int_{0}^{t}\left(\mathcal{A}_{n} u_{n}(s), v\right) d s=\left(x_{0}, v\right)+\int_{0}^{t}\left(\mathscr{B}_{n}\left(u_{n}(s)\right), v\right) d s \\
& +\int_{0}^{t}\left(f_{n}(s), v\right) d s+\int_{0}^{t}\left(\mathfrak{C}_{n} u_{n}(s), v\right) d w(s),
\end{aligned}
$$

for all $v \in H_{n}, t \in[0, T]$ and a.e. $\omega \in \Omega$.

Theorem 2.1: For each $n \geq 1$, equation $\left(P_{n}\right)$ has a solution $u_{n} \in \mathcal{L}_{V}^{2}(\Omega \times[0, T])$, which is unique almost surely and has in $H$ almost surely continuous trajectories.

Sketch of Proof: Let $\left(\chi_{n}\right)$ be a family of Lipschitz continuous mappings such that

$$
\chi_{n}(x)=\left\{\begin{array}{cl}
1, & \text { if } 0 \leq x \leq n, \\
0, & \text { if } x \geq n+1, \\
n+1-x, & \text { if } x \in(n, n+1) .
\end{array}\right.
$$

For each fixed $n$ we consider the solution $u_{n}$ of equation $\left(P_{n}\right)$ approximated by $u_{n}^{M}(M=1,2, \ldots)$, which is a solution of the equation

$$
\begin{gathered}
\left(u_{n}^{M}(t), v\right)+\int_{0}^{t}\left(\mathcal{A}_{n} u_{n}^{M}(s), v\right) d s=\left(x_{0}, v\right) \\
+\int_{0}^{t}\left(\chi_{M}\left(\left\|u_{n}^{M}(t)\right\|^{2}\right) \mathscr{B}_{n}\left(u_{n}^{M}(s), u_{n}^{M}(s)\right), v\right) d s+\int_{0}^{t}\left(f_{n}(s), v\right) d s \\
+\int_{0}^{t}\left(\mathbb{C}_{n} u_{n}^{M}(s), v\right) d w(s)
\end{gathered}
$$

for all $v \in H_{n}, t \in[0, T]$ and a.e. $\omega \in \Omega$. For this equation, we can apply the theory of finite dimensional Itô equations with Lipschitz continuous nonlinearities (see [9, Theorem 2.9, p. 289]). Then one can prove that for $M \rightarrow \infty$, the sequence $\left(u_{n}^{M}\right)$ $(M=1,2, \ldots)$ converges in probability to $u_{n}$; for more details we refer the reader to [15, Lemma 2.3.1, pp. 21-23]). The uniqueness of the solution can be proved analogously to the case of the stochastic Navier-Stokes equation (see Lemma 3.4).

The purpose of this paper is to prove that the sequence $\left(u_{n}\right)$ approximates the solution $u$ of the Navier-Stokes equation in mean square.

The main results of this paper are given in the following two theorems.

Theorem 2.2: Equation (2) has a solution in the space $\mathcal{L}_{V}^{2}(\Omega \times[0, T])$. The solution is unique almost surely and has in $H$ almost surely continuous trajectories.

This theorem is proved by using Lemma 3.1- Lemma 3.4.

Theorem 2.3: The following convergences hold:

$$
E \int_{0}^{T}\left\|u(s)-u_{n}(s)\right\|_{V}^{2} d s \rightarrow 0 \text { for } n \rightarrow \infty
$$


and for all $t \in[0, T]$

$$
E\left\|u(t)-u_{n}(t)\right\|^{2} \rightarrow 0 \text { for } n \rightarrow \infty \text {. }
$$

This theorem will be proved in the final part of Section 3 .

Remarks: 1) The sequence $\left(u_{n}\right)$ represents the Galerkin approximation for the strong solution of the Navier-Stokes equation

2) Since $\mathcal{A}$ is a linear and monotone operator, it follows that it is continuous (see [19, Proposition 26.4 , p. 555]), i.e., there exists a constant $c_{\mathcal{A}}>0$ such that for all $u \in V$

$$
\|\mathcal{A} u\|_{V^{*}}^{2} \leq c_{\mathcal{A}}\|u\|_{V}^{2}
$$

3) The condition $\mathrm{CO}=0$ is given only to simplify the calculations. It can be omitted, in which case one can use the estimate $\|\mathrm{C} u\|^{2} \leq 2 \lambda\|u\|^{2}+2\|\mathrm{C} 0\|^{2}$ that follows from the Lipschitz condition.

4) If we set $n=2, V=\left\{u \in \stackrel{\circ}{W}_{2}^{1}(G): \operatorname{div} u=0\right\}, H=\bar{V}^{L^{2}(G)}$ and

$$
\langle\mathcal{A} u, v\rangle=\int_{G} \sum_{i=1}^{n} \frac{\partial u}{\partial x_{i}} \frac{\partial v}{\partial x_{i}} d x,\langle\mathscr{B}(u, v), z\rangle=-\int_{G} \sum_{i, j=1}^{n} u_{i} \frac{\partial v_{j}}{\partial x_{i}} z_{j} d x
$$

for $u, v, z \in V$, then equation (1) can be transformed into (2); see [16].

\section{Proof of the Main Result}

We need several lemmas.

\section{Lemma 3.1:}

(i) There exists a positive constant $c_{1}$ such that for all $n=1,2, \ldots$

$$
E\left\|u_{n}(T)\right\|^{2}+2 \nu E \int_{0}^{T}\left\|u_{n}(t)\right\|_{V}^{2} d t \leq c_{1}\left[E\left\|x_{0}\right\|^{2}+E \int_{0}^{T}\|f(t)\|^{2} d t\right]
$$

and each of the following expressions

$$
\sup _{t \in[0, T]} E\left\|u_{n}(t)\right\|^{4}, E \int_{0}^{T}\left\|u_{n}(t)\right\|_{V}^{2}\left\|u_{n}(t)\right\|^{2} d t, E\left(\int_{0}^{T}\left\|u_{n}(t)\right\|_{V}^{2} d t\right)^{2}
$$

is less or equal than $c_{1}\left[E\left\|x_{0}\right\|^{4}+E \int_{0}^{T}\|f(t)\|^{4} d t\right]$.

(ii) There exist $u \in \mathcal{L}_{V}^{2}\left(\Omega \times[0, T], \Re^{*} \in \mathcal{L}_{V^{*}}^{2}(\Omega \times[0, T]), \mathrm{C}^{*} \in \mathcal{L}_{H}^{2}(\Omega \times[0, T])\right.$ and a subsequence $\left(n^{\prime}\right)$ of $(n)$ such that for $n^{\prime} \rightarrow \infty$ we have

$$
\begin{gathered}
u_{n^{\prime}} \rightarrow u \text { in } \ell_{V}^{2}(\Omega \times[0, T]), \\
\mathscr{B}\left(u_{n^{\prime}}, u_{n^{\prime}}\right) \rightarrow \mathscr{B}^{*} \text { in } \mathcal{L}_{V^{*}}^{2}(\Omega \times[0, T]),
\end{gathered}
$$




$$
\mathrm{e} u_{n^{\prime}} \rightarrow \mathrm{e}^{*} \text { in } \ell_{H}^{2}(\Omega \times[0, T])
$$

(iii) For all $v \in V, t \in[0, T]$ and a.e. $\omega \in \Omega$ the process $(u(t))_{t \in[0, T]}$ satisfies the equation:

$$
\begin{aligned}
(u(t), v) & +\int_{0}^{t}\langle\mathcal{A} u(s), v\rangle d s=\left(x_{0}, v\right)+\int_{0}^{t}\left\langle\mathfrak{B}^{*}(s), v\right\rangle d s \\
& +\int_{0}^{t}(f(s), v) d s+\int_{0}^{t}\left(\mathrm{C}^{*}(s), v\right) d w(s) .
\end{aligned}
$$

The process $(u(t))_{t \in[0, T]}$ has in $H$ almost surely continuous trajectories.

Proof: $(i)$ Let $n$ be an arbitrary fixed natural number. Equation $\left(P_{n}\right)$ can also be written as

$$
\begin{aligned}
\left(u_{n}(t), h_{i}\right)+ & \int_{0}^{t}\left\langle\mathcal{A} u_{n}(s), h_{i}\right\rangle d s=\left(x_{0}, h_{i}\right)+\int_{0}^{t}\left\langle\mathfrak{B}\left(u_{n}(s), u_{n}(s)\right), h_{i}\right\rangle d s \\
& +\int_{0}^{t}\left(f(s), h_{i}\right) d s+\int_{0}^{t}\left(\mathcal{C} u_{n}(s), h_{i}\right) d w(s)
\end{aligned}
$$

for $i=1, \ldots, n, t \in[0, T]$ and a.e. $\omega \in \Omega$.

Let $z(t)=\exp \{-(6 \lambda+3) t\}$, so $z(t)=1-\int_{0}^{t}(6 \lambda+3) z(s) d s$ for each $t \in[0, T]$. From (4), the Itô formula, and hypothesis (iii) we have

$$
\begin{gathered}
z(t)\left\|u_{n}(t)\right\|^{2}+2 \int_{0}^{t} z(s)\left\langle\mathcal{A} u_{n}(s), u_{n}(s)\right\rangle d s=\left\|x_{0}\right\|^{2}+2 \int_{0}^{t} z(s)\left(f(s), u_{n}(s)\right) d s \\
+\int_{0}^{t} z(s)\left\|\mathrm{C} u_{n}(s)\right\|^{2} d s-(6 \lambda+3) \int_{0}^{t} z(s)\left\|u_{n}(s)\right\|^{2} d s \\
+2 \int_{0}^{t} z(s)\left(\mathcal{C} u_{n}(s), u_{n}(s)\right) d w(s)
\end{gathered}
$$

and

$$
\begin{gathered}
z(t)\left\|u_{n}(t)\right\|^{4}+4 \int_{0}^{t} z(s)\left\langle\mathcal{A} u_{n}(s), u_{n}(s)\right\rangle\left\|u_{n}(s)\right\|^{2} d s=4 \int_{0}^{t} z(s)\left(\mathcal{C} u_{n}(s), u_{n}(s)\right)^{2} d s \\
+2 \int_{0}^{t} z(s)\left\|\mathrm{C} u_{n}(s)\right\|^{2}\left\|u_{n}(s)\right\|^{2} d s-(6 \lambda+3) \int_{0}^{t} z(s)\left\|u_{n}(s)\right\|^{4} d s \\
+4 \int_{0}^{t} z(s)\left(f(s), u_{n}(s)\right)\left\|u_{n}(s)\right\|^{2} d s
\end{gathered}
$$




$$
+4 \int_{0}^{t} z(s)\left(\mathcal{C} u_{n}(s), u_{n}(s)\right)\left\|u_{n}(s)\right\|^{2} d w(s)+\left\|x_{0}\right\|^{4}
$$

Using the properties of $\mathcal{A}, \mathcal{C}$ and some elementary calculations, we obtain

$$
\begin{gathered}
z(t)\left\|u_{n}(t)\right\|^{2}+2 \nu \int_{0}^{t} z(s)\left\|u_{n}(s)\right\|_{V}^{2} d s \\
\leq\left\|x_{0}\right\|^{2}+\int_{0}^{t} z(s)\|f(s)\|^{2} d s+2 \int_{0}^{t} z(s)\left(\mathrm{C} u_{n}(s), u_{n}(s)\right) d w(s)
\end{gathered}
$$

and$$
z(t)\left\|u_{n}(t)\right\|^{4}+4 \nu \int_{0}^{t} z(s)\left\|u_{n}(s)\right\|_{V}^{2}\left\|u_{n}(s)\right\|^{2} d s
$$$$
\leq\left\|x_{0}\right\|^{4}+\int_{0}^{t} z(s)\|f(s)\|^{4} d s+4 \int_{0}^{t} z(s)\left(\mathcal{C} u_{n}(s), u_{n}(s)\right)\left\|u_{n}(s)\right\|^{2} d w(s) .
$$

In (7) we square both sides of the inequality, use some well-known inequalities and obtain

$$
\begin{gathered}
z^{2}(t)\left\|u_{n}(t)\right\|^{4}+4 \nu^{2}\left(\int_{0}^{t} z(s)\left\|u_{n}(s)\right\|_{V}^{2} d s\right)^{2} \\
\leq 3\left\|x_{0}\right\|^{4}+3\left(\int_{0}^{t} z(s)\|f(s)\|^{2} d s\right)^{2}+12\left|\int_{0}^{t} z(s)\left(\mathcal{C} u_{n}(s), u_{n}(s)\right) d w(s)\right|^{2} .
\end{gathered}
$$

Taking the expectation in (7), (8) and (9) we obtain

$$
\begin{gathered}
E z(t)\left\|u_{n}(t)\right\|^{2}+2 \nu E \int_{0}^{t} z(s)\left\|u_{n}(s)\right\|_{V}^{2} d s \leq E\left\|x_{0}\right\|^{2}+E \int_{0}^{t} z(s)\|f(s)\|^{2} d s \\
E z(t)\left\|u_{n}(t)\right\|^{4}+4 \nu E \int_{0}^{t} z(s)\left\|u_{n}(s)\right\|_{V}^{2}\left\|u_{n}(s)\right\|^{2} d s \\
\leq E\left\|x_{0}\right\|^{2}+E \int_{0}^{t} z(s)\|f(s)\|^{4} d s
\end{gathered}
$$

and

$$
\begin{gathered}
E z^{2}(t)\left\|u_{n}(t)\right\|^{4}+4 \nu^{2} E\left(\int_{0}^{t} z(s)\left\|u_{n}(s)\right\|_{V}^{2} d s\right)^{2} \\
\leq 3 E\left\|x_{0}\right\|^{4}+3 E\left(\int_{0}^{t} z(s)\|f(s)\|^{2} d s\right)^{2}+12 E\left|\int_{0}^{t} z(s)\left(\mathrm{C} u_{n}(s), u_{n}(s)\right) d w(s)\right|^{2} .
\end{gathered}
$$


From the properties of $z$ it then follows that

$$
\begin{gathered}
E\left\|u_{n}(T)\right\|^{2}+2 \nu E \int_{0}^{T}\left\|u_{n}(s)\right\|_{V}^{2} d s \leq \exp \{(6 \lambda+3) T\}\left[E\left\|x_{0}\right\|^{2}+E \int_{0}^{T}\|f(s)\|^{2} d s\right], \\
\sup _{t \in[0, T]} E\left\|u_{n}(t)\right\|^{4}+4 \nu E \int_{0}^{T}\left\|u_{n}(s)\right\|_{V}^{2}\left\|u_{n}(s)\right\|^{2} d s \\
\leq \exp \left\{(6 \lambda+3 T\}\left[E\left\|x_{0}\right\|^{4}+E \int_{0}^{T}\|f(s)\|^{4} d s\right] .\right.
\end{gathered}
$$

In (10) we apply the property of the second moment of the Ito integral and the Schwartz inequality to obtain

$$
\begin{gathered}
E z^{2}(t)\left\|u_{n}(t)\right\|^{4}+4 \nu^{2} E\left(\int_{0}^{t} z(s)\left\|u_{n}(s)\right\|_{V}^{2} d s\right)^{2} \\
\leq 3 E\left\|x_{0}\right\|^{4}+3 t E \int_{0}^{t} z^{2}(s)\|f(s)\|^{4} d s+12 E \int_{0}^{t} z^{2}(s)\left(C u_{n}(s), u_{n}(s)\right)^{2} d s \\
\leq 3 E\left\|x_{0}\right\|^{4}+3 t E \int_{0}^{t}\|f(s)\|^{4} d s+12 \lambda E \int_{0}^{t}\left\|u_{n}(s)\right\|^{4} d s .
\end{gathered}
$$

From (11) and (12) it follows that there exists a constant $c_{1}$ (depending on $\lambda$ and $T$ ) such that

$$
\begin{gathered}
E\left\|u_{n}(T)\right\|^{2}+2 \nu E \int_{0}^{T}\left\|u_{n}(s)\right\|_{V}^{2} d s \leq c_{1}\left[E\left\|x_{0}\right\|^{2}+E \int_{0}^{T}\|f(s)\|^{2} d s\right] \\
\sup _{t \in[0, T]} E\left\|u_{n}(t)\right\|^{4}+4 \nu E \int_{0}^{T}\left\|u_{n}(s)\right\|_{V}^{2}\left\|u_{n}(s)\right\|^{2} d s \\
\leq c_{1}\left[E\left\|x_{0}\right\|^{4}+E \int_{0}^{T}\|f(s)\|^{4} d s\right]
\end{gathered}
$$

and

$$
E\left(\int_{0}^{T}\left\|u_{n}(s)\right\|_{V}^{2} d s\right)^{2} \leq c_{1}\left[E\left\|x_{0}\right\|^{4}+E \int_{0}^{T}\|f(s)\|^{4} d s\right] .
$$

(ii) The right-hand side of inequality (13) does not depend on $n$. Taking into consideration the properties of $C$ and the estimate from the first part of the proof of this lemma, it follows that $\left(\mathcal{C} u_{n}\right)$ is a bounded sequence in the space $\mathcal{L}_{H}^{2}(\Omega \times[0, T])$. 
From $(i)$ and the properties of $\mathfrak{B}$ we can derive

$$
\begin{gathered}
E \int_{0}^{T}\left\|\mathfrak{B}\left(u_{n}(t), u_{n}(t)\right)\right\|_{V^{*}}^{2} d t \leq b E \int_{0}^{T}\left\|u_{n}(t)\right\|_{V}^{2}\left\|u_{n}(t)\right\|^{2} d t \\
\leq \frac{b c_{1}}{4 \nu}\left[E\left\|x_{0}\right\|^{4}+E \int_{0}^{T}\|f(s)\|^{4} d s\right]
\end{gathered}
$$

so $\left(\mathscr{B}\left(u_{n}, u_{n}\right)\right)$ is a bounded sequence in the space $\mathcal{L}_{V^{*}}^{2}(\Omega \times[0, T])$. Applying the weakly sequentially compactness property (see [18, Proposition 10.13, p. 480]), it follows that there exist a subsequence $\left(n^{\prime}\right)$ of $(n)$ and $\widehat{u} \in \mathcal{L}_{V}^{2}(\Omega \times[0, T])$, $\mathfrak{B}^{*} \in$ $\mathcal{L}_{V^{*}}^{2}(\Omega \times[0, T]), \mathrm{C}^{*} \in \mathcal{L}_{H}^{2}(\Omega \times[0, T])$ such that for $n^{\prime} \rightarrow \infty$

$$
\begin{gathered}
u_{n^{\prime}} \rightarrow \widehat{u} \text { in } \mathcal{L}_{V}^{2}(\Omega \times[0, T]), \mathscr{B}\left(u_{n^{\prime}}, u_{n^{\prime}}\right) \rightarrow \mathscr{B}^{*} \text { in } \mathcal{L}_{V^{*}}^{2}(\Omega \times[0, T]), \\
\mathcal{e} u_{n^{\prime}} \rightarrow \mathcal{C}^{*} \text { in } \mathcal{L}_{H}^{2}(\Omega \times[0, T]) .
\end{gathered}
$$

(iii) In (4) we take $n^{\prime} \rightarrow \infty$, use the properties of $\mathcal{A}$, the weak convergences from above (also see Corollary 4.3 from the Appendix) to arrive at

$$
\begin{aligned}
\left(\widehat{u}(t), h_{i}\right) & =\left(x_{0}, h_{i}\right)-\int_{0}^{t}\left\langle\mathcal{A} \widehat{u}(s), h_{i}\right\rangle d s+\int_{0}^{t}\left\langle\mathfrak{B}^{*}(s), h_{i}\right\rangle d s \\
& +\int_{0}^{t}\left(f(s), h_{i}\right) d s+\int_{0}^{t}\left(\mathcal{C}^{*}(s), h_{i}\right) d w(s),
\end{aligned}
$$

for a.e. $(\omega, t) \in \Omega \times[0, T]$ and $i$ an arbitrary fixed natural number. Since $\operatorname{sp}\left\{h_{1}, \ldots\right.$, $\left.h_{n}, \ldots\right\} \subset V$ is dense in $V$ (because of the properties of the eigenvectors of $\mathcal{A}$ ) it follows that (14) holds also for all $v \in V$.

From the Itô formula for $\|\cdot\|^{2}$ applied to processes over $\left(V, H, V^{*}\right)$, it follows that there exists a $\mathcal{F}_{t}$-measurable $H$-valued process, which is also equal to $\widehat{u}(t)$ (for a.e. $(\omega, t) \in \Omega \times[0, T])$ and is equal to the right-hand side of (14) for all $t \in[0, T]$ and

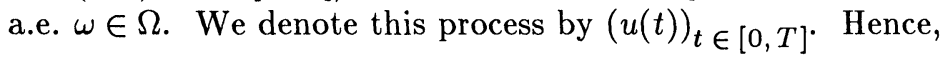

$$
\begin{aligned}
& (u(t), v)+\int_{0}^{t}\langle\mathcal{A} u(s), v\rangle d s \\
& \quad=\left(x_{0}, v\right)+\int_{0}^{t}\left\langle\mathfrak{B}^{*}(s), v\right\rangle d s+\int_{0}^{t}(f(s), v) d s+\int_{0}^{t}\left(\mathrm{C}^{*}(s), v\right) d w(s)
\end{aligned}
$$

for all $v \in V, t \in[0, T]$ and a.e. $\omega \in \Omega$; the process $(u(t))_{t \in[0, T]}$ has in $H$ almost surely continuous trajectories (see [11, Theorem 3.1, p. 88]).

For each natural number $M$, let us introduce the following stopping times: 


$$
\begin{gathered}
\tau_{M}^{1}=\left\{\begin{array}{cl}
T, & \text { if } \sup _{t \in[0, T]}\|u(t)\|^{2}<M \\
\inf \left\{t \in[0, T]:\|u(t)\|^{2} \geq M\right\}, & \text { otherwise, }
\end{array}\right. \\
\tau_{M}^{2}=\left\{\begin{array}{cl}
T, & \text { if } \int_{0}^{T}\|u(s)\|_{V}^{2} d s<M \\
\inf \left\{t \in[0, T]: \int_{0}^{t}\|u(s)\|_{V}^{2} d s \geq M\right\}, & \text { otherwise. }
\end{array}\right.
\end{gathered}
$$

Let

$$
\tau_{M}:=\min \left\{\tau_{M}^{1}, \tau_{M}^{2}\right\}
$$

Since $u$ has continuous trajectories in $H$, we see that for all $t \in[0, T]$,

$$
\mid u\left(t \wedge \tau_{M}\right)\left\|^{2} \leq M, \int_{0}^{t \wedge \tau_{M}}\right\| u(s) \|_{V}^{2} d s \leq M \text { for a.e. } \omega \in \Omega .
$$

Lemma 3.2: (i) The following convergences hold:

$$
\lim _{M \rightarrow \infty} P\left(\tau_{M}<T\right)=0
$$

and for a.e. $\omega \in \Omega$

$$
\lim _{M \rightarrow \infty} \tau_{M}=T
$$

(ii) The function $u$ from Lemma 3.1 satisfies

$$
E \sup _{t \in[0, T]}\|u(t)\|^{2}<\infty
$$

Proof: (i) From Lemma 3.1 we have

$$
\sup _{t \in[0, T]}\|u(t)\|^{2}<\infty, \quad \int_{0}^{T}\|u(s)\|^{2} d s<\infty \text { for a.e. } \omega \in \Omega
$$

Using some elementary inequalities and arguments we obtain

$$
\begin{gathered}
\lim _{M \rightarrow \infty} P\left(\tau_{M}<T\right) \leq \lim _{M \rightarrow \infty} P\left(\tau_{M}^{1}<T\right)+\lim _{M \rightarrow \infty} P\left(\tau_{M}^{2}<T\right) \\
\leq \lim _{M \rightarrow \infty} P\left(\sup _{t \in[0, T]}\|u(t)\|^{2} \geq M\right)+\lim _{M \rightarrow \infty}\left(P \int_{0}^{T}\|u(s)\|_{V}^{2} d s \geq M\right) \\
\leq P\left(\bigcap_{M=1}^{\infty}\left(\sup _{t \in[0, T]}\|u(t)\|^{2} \geq M\right)\right)+P\left(\bigcap_{M=1}^{\infty}\left(\int_{0}^{T}\|u(s)\|_{V}^{2} \geq M\right)\right)=0 .
\end{gathered}
$$

The sequence $\left(T-\tau_{M}\right)_{M}$ is monotone decreasing (for a.e. $\omega \in \Omega$ ) and above we have proved that it converges in probability to zero. Therefore, it converges to zero for almost every $\omega \in \Omega$. 
(ii) In (3) we apply the Itô formula and take $t=\tau_{M}$, use the Burkholder inequality (see $[9$, p. 166]) and some elementary inequalities, and obtain

$$
\begin{aligned}
& E \sup _{t \in\left[0, \tau_{M}\right]}\|u(t)\|^{2} \leq E\left\|x_{0}\right\|^{2}+2 E \int_{0}^{\tau_{M}}\left\|\Re^{*}(s)\right\|_{V^{*}}\|u(s)\|_{V^{d}} d s \\
& +2 E \int_{0}^{\tau_{M}}\|f(s)\|\|u(s)\| d s+2 E \sup _{t \in\left[0, \tau_{M}\right]}\left|\int_{0}^{t}\left(\mathcal{C}^{*}(s), u(s)\right) d w(s)\right| \\
& +E \int_{0}^{\tau_{M}}\left\|\mathrm{C}^{*}(s)\right\|^{2} d s \leq E\left\|x_{0}\right\|^{2} \\
& +E \int_{0}^{\tau}\left(\left\|\mathscr{B}^{*}(s)\right\|_{V^{*}}^{2}+\|u(s)\|_{V}^{2}+\|u(s)\|^{2}+\|f(s)\|^{2}+\left\|\mathrm{C}^{*}(s)\right\|^{2}\right) d s \\
& +2 k_{1} E\left(\sup _{t \in\left[0, \tau_{M}\right]}\|u(t)\|^{2} \int_{0}^{\tau_{M}}\left\|e^{*}(s)\right\|^{2} d s\right)^{\frac{1}{2}} \\
& \leq E\left\|x_{0}\right\|^{2}+\frac{1}{2} E \sup _{t \in\left[0, \tau_{M}\right]}\|u(t)\|^{2} \\
& +E \int_{0}^{\tau}\left(\left\|\mathscr{B}^{*}(s)\right\|_{V^{*}}^{2}+\|u(s)\|_{V}^{2}+\|u(s)\|^{2}+\|f(s)\|^{2}+k_{2}\left\|\mathcal{C}^{*}(s)\right\|^{2}\right) d s
\end{aligned}
$$

where $k_{1}, k_{2}$ are positive constants $\left(k_{1}\right.$ is the constant that occurs in the Burkholder inequality). We get

$$
\begin{aligned}
& \frac{1}{2} E \sup _{t \in\left[0, \tau_{M}\right]}\|u(t)\|^{2} \leq E\left\|x_{0}\right\|^{2} \\
& \quad+E \int_{0}^{\tau_{M}}\left(\left\|\mathscr{B}^{*}(s)\right\|_{V^{*}}^{2}+\|u(s)\|_{V}^{2}+\|u(s)\|^{2}+\|f(s)\|^{2}+k_{2}\left\|\mathcal{C}^{*}(s)\right\|^{2}\right) d s .
\end{aligned}
$$

Since $\tau_{M} \rightarrow T$ for a.e. $\omega \in \Omega$ it follows that

$$
\begin{aligned}
& \frac{1}{2} E \sup _{t \in[0, T]}\|u(t)\|^{2} \leq E\left\|x_{0}\right\|^{2} \\
& \quad+E \int_{0}^{T}\left(\left\|\mathfrak{B}^{*}(s)\right\|_{V^{*}}^{2}+\|u(s)\|_{V}^{2}+\|u(s)\|^{2}+\|f(s)\|^{2}+k_{2}\left\|\mathrm{C}^{*}(s)\right\|^{2}\right) d s .
\end{aligned}
$$

But $u \in \mathcal{L}_{V}^{2}(\Omega \times[0, T]), \mathfrak{B}^{*} \in \mathcal{L}_{V^{*}}^{2}(\Omega \times[0, T]), \mathrm{e}^{*}, f \in \mathcal{L}_{H}^{2}(\Omega \times[0, T])$. Consequently, 


$$
E \sup _{t \in[0, T]}\|u(t)\|^{2}<\infty
$$

Lemma 3.3: For each fixed natural number $M$,

and

$$
E \int_{0}^{\tau}\left\|u(s)-u_{n^{\prime}}(s)\right\|_{V}^{2} d s \rightarrow 0 \text { for } n^{\prime} \rightarrow \infty
$$

$$
E\left\|u\left(\tau_{M}\right)-u_{n^{\prime}}\left(\tau_{M}\right)\right\|^{2} \rightarrow 0 \text { for } n^{\prime} \rightarrow \infty
$$

Proof: For each natural number $n$, let $\tilde{u}_{n} \in \mathcal{L}_{H_{n}}^{2}(\Omega \times[0, T])$ be defined by

$$
\tilde{u}_{n}(t)=\sum_{i=1}^{n}\left(u(t), h_{i}\right) h_{i}
$$

for all $t \in[0, T]$ and a.e. $\omega \in \Omega$. Using the properties of $\mathcal{A}$ and of its eigenvectors $\left\{h_{1}, h_{2}, \ldots\right\}\left(\left\{\lambda_{1}, \lambda_{2}, \ldots\right\}\right.$ are the corresponding eigenvalues $)$, we have

$$
\begin{gathered}
\left\|\tilde{u}_{n}(t)\right\|^{2} \leq\|u(t)\|^{2}, \quad\left\|u(t)-\tilde{u}_{n}(t)\right\|^{2} \leq\|u(t)\|^{2} \\
\nu\left\|u(t)-\tilde{u}_{n}(t)\right\|_{V}^{2} \leq\left\langle\mathcal{A} u(t)-\mathcal{A} \tilde{u}_{n}(t), u(t)-\tilde{u}_{n}(t)\right\rangle \\
=\sum_{i=n}^{\infty} \lambda_{i}\left(u(t), h_{i}\right)^{2} \leq\langle\mathcal{A} u(t), u(t)\rangle \leq c_{\mathcal{A}}\|u(t)\|_{V}^{2}
\end{gathered}
$$

Hence for all $t \in[0, T]$ and a.e. $\omega \in \Omega$,

$$
\left\|u(t)-\tilde{u}_{n}(t)\right\|_{V}^{2} \rightarrow 0 \text { for } n \rightarrow \infty
$$

By the Lebesgue Dominated Convergence Theorem, it follows that

$$
E \int_{0}^{T}\left\|u(t)-\tilde{u}_{n}(t)\right\|_{V}^{2} d t \rightarrow 0 \text { for } n \rightarrow \infty
$$

From (3) and (4) we have

$$
\begin{gathered}
\left(u(t)-u_{n}(t), h_{i}\right)+\int_{0}^{t}\left\langle\mathcal{A} u(s)-\mathcal{A} u_{n}(s), h_{i}\right\rangle d s \\
=\int_{0}^{t}\left\langle\mathfrak{B}^{*}(s)-\mathscr{B}\left(u_{n}(s), u_{n}(s)\right), h_{i}\right\rangle d s+\int_{0}^{t}\left(\mathcal{C}^{*}(s)-\mathcal{C} u_{n}(s), h_{i}\right) d w(s),
\end{gathered}
$$

for all $t \in[0, T], i=1, \ldots, n$, a.e. $\omega \in \Omega$. After applying the Itô formula and summing $i$ from 1 to $n$, we use the properties of $\mathcal{A}$ to obtain

$$
\left\|\tilde{u}_{n}(t)-u_{n}(t)\right\|^{2}+2 \int_{0}^{t}\left\langle\mathcal{A} \tilde{u}_{n}(s)-\mathcal{A} u_{n}(s), \tilde{u}_{n}(s)-u_{n}(s)\right\rangle d s
$$




$$
\begin{gathered}
=2 \int_{0}^{t}\left\langle\mathfrak{B}^{*}(s)-\mathscr{B}\left(u_{n}(s), u_{n}(s)\right), \tilde{u}_{n}(s)-u_{n}(s)\right\rangle d s \\
+2 \int_{0}^{t}\left(\mathcal{C}^{*}(s)-\mathcal{C} u_{n}(s), \tilde{u}_{n}(s)-u_{n}(s)\right) d w(s)+\int_{0}^{t} \sum_{i=1}^{n}\left(\mathcal{C}^{*}(s)-\mathcal{C} u_{n}(s), h_{i}\right)^{2} d s
\end{gathered}
$$

for all $t \in[0, T], i=1, \ldots, n$, a.e. $\omega \in \Omega$. Write

$$
y(t)=\exp \left\{-\frac{b}{\nu} \int_{0}^{t}\|u(s)\|_{V}^{2} d s-\lambda t\right\}
$$

for all $t \in[0, T]$ and a.e. $\omega \in \Omega$. By the Itô formula we have

$$
\begin{gathered}
y(t)\left\|\tilde{u}_{n}(t)-u_{n}(t)\right\|^{2}+2 \int_{0}^{t} y(s)\left\langle\mathcal{A} \tilde{u}_{n}(s)-\mathcal{A} u_{n}(s), \tilde{u}_{n}(s)-u_{n}(s)\right\rangle d s \\
=2 \int_{0}^{t} y(s)\left\langle\mathfrak{B}^{*}(s)-\mathfrak{B}\left(u_{n}(s), u_{n}(s)\right), \tilde{u}_{n}(s)-u_{n}(s)\right\rangle d s \\
-\frac{b}{\nu} \int_{0}^{t} y(s)\|u(s)\|_{V}^{2}\left\|\tilde{u}_{n}(s)-u_{n}(s)\right\|^{2} d s-\lambda \int_{0}^{t} y(s)\left\|\tilde{u}_{n}(s)-u_{n}(s)\right\|^{2} d s \\
+\int_{0}^{t} \sum_{i=1}^{n} y(s)\left(\mathbb{C}^{*}(s)-\mathrm{Cu}_{n}(s), h_{i}\right)^{2} d s+2 \int_{0}^{t} y(s)\left(\mathbb{C}^{*}(s)-\mathrm{C} u_{n}(s), \tilde{u}_{n}(s)-u_{n}(s)\right) d w(s)
\end{gathered}
$$

for all $t \in[0, T], i=1, \ldots, n$, a.e. $\omega \in \Omega$. From the properties of $\mathscr{B}$ and $\tilde{u}_{n}$ we see that

$$
\begin{gathered}
\left\langle\mathscr{B}\left(u_{n}, u_{n}\right), \tilde{u}_{n}-u_{n}\right\rangle=\left\langle\mathscr{B}\left(u_{n}, \tilde{u}_{n}\right), \tilde{u}_{n}-u_{n}\right\rangle \\
=\left\langle\mathscr{B}\left(u_{n}-\tilde{u}_{n}, \tilde{u}_{n}\right), \tilde{u}_{n}-u_{n}\right\rangle+\left\langle\mathscr{B}\left(\tilde{u}_{n}, \tilde{u}_{n}\right), \tilde{u}_{n}-u_{n}\right\rangle \\
\leq \frac{b}{2 \nu}\left\|\tilde{u}_{n}\right\|_{V}^{2}\left\|\tilde{u}_{n}-u_{n}\right\|^{2}+\frac{\nu}{2}\left\|\tilde{u}_{n}-u_{n}\right\|_{V}^{2}+\left\langle\mathscr{B}\left(\tilde{u}_{n}, \tilde{u}_{n}\right), \tilde{u}_{n}-u_{n}\right\rangle \\
\leq \frac{b}{2 \nu}\|u\|_{V}^{2}\left\|\tilde{u}_{n}-u_{n}\right\|^{2}+\frac{\nu}{2}\left\|\tilde{u}_{n}-u_{n}\right\|_{V}^{2}+\left\langle\mathscr{B}\left(\tilde{u}_{n}, \tilde{u}_{n}\right), \tilde{u}_{n}-u_{n}\right\rangle .
\end{gathered}
$$

From the properties of $\mathcal{C}$ and $\tilde{u}_{n}$ we get

$$
\begin{gathered}
\sum_{i=1}^{n}\left(\mathrm{C}^{*}(s)-\mathrm{C} u_{n}(s), h_{i}\right)^{2}=\left\|\mathrm{C} u(s)-\mathrm{C} u_{n}(s)\right\|_{H_{n}}^{2} \\
\left.+2\left(\mathrm{C}^{*}(s)-\mathrm{C} u_{n}(s), \mathrm{C}^{*}(s)-\mathrm{C} u(s)\right)_{H_{n}}-\| \mathrm{C} u(s)-\mathrm{C}^{*}(s)\right) \|_{H_{n}}^{2} \\
\leq \lambda\left\|u(s)-\tilde{u}_{n}(s)\right\|^{2}+\lambda\left\|\tilde{u}_{n}(s)-u_{n}(s)\right\|^{2}+2\left(\mathrm{C}^{*}(s)-\mathrm{C} u_{n}(s), \mathrm{C}^{*}(s)-\mathrm{C} u(s)\right)_{H_{n}}
\end{gathered}
$$




$$
-\left\|\mathrm{C} u(s)-\mathrm{C}^{*}(s)\right\|_{H_{n}}^{2}
$$

where $(x, y)_{H_{n}}:=\sum_{i=1}^{n}\left(x, h_{i}\right)\left(y, h_{i}\right)$ for all $x, y \in H_{n}=\operatorname{sp}\left\{h_{1}, h_{2}, \ldots, h_{n}\right\}$.

We use these estimates in $(*)$ to get

$$
\begin{gathered}
E y\left(\tau_{M}\right)\left\|\tilde{u}_{n}\left(\tau_{M}\right)-u_{n}\left(\tau_{M}\right)\right\|^{2}+\frac{3 \nu}{2} E \int_{0}^{\tau_{M}} y(s)\left\|\tilde{u}_{n}(s)-u_{n}(s)\right\|_{V}^{2} d s \\
\quad+E \int_{0}^{\tau_{M}} y(s)\left\|\mathrm{C} u(s)-\mathrm{C}^{*}(s)\right\|_{H_{n}^{2}}^{2} d s \\
\leq 2 E \int_{0}^{\tau_{M}} y(s)\left\langle\mathscr{B}^{*}(s)-\mathscr{B}\left(\tilde{u}_{n}(s), \tilde{u}_{n}(s)\right), \tilde{u}_{n}(s)-u_{n}(s)\right\rangle d s \\
+\lambda E \int_{0}^{\tau_{M}} y(s)\left\|u(s)-\tilde{u}_{n}(s)\right\|^{2} d s+2 E \int_{0}^{\tau_{M}} y(s)\left(\mathrm{C}^{*}(s)-\mathrm{C} u_{n}(s), \mathrm{C}^{*}(s)-\mathrm{Cu}(s)\right)_{H_{n}} d s
\end{gathered}
$$

where $M$ is an arbitrary fixed real number. Using the properties of $\mathscr{B}$, those of the stopping time $\tau_{M}$, and the fact that $\left(\tilde{u}_{n}\right)_{n}$ is the partial sum of the Fourier expansion of $u \in \mathcal{L}_{V}^{2}(\Omega \times[0, T]$ ) (see the properties given in (16) and (17)) we have

$$
\begin{gathered}
E \int_{0}^{\tau_{M}} y(s)\left\|\mathscr{B}(u(s), u(s))-\mathfrak{B}\left(\tilde{u}_{n}(s), \tilde{u}_{n}(s)\right)\right\|_{V^{*}}^{2} d s \\
\leq E \int_{0}^{\tau_{M}} y(s)\left(\|u(s)\|_{V}\|u(s)\|+\left\|\tilde{u}_{n}(s)\right\|_{V}\left\|\tilde{u}_{n}(s)\right\|\right)\left\|u(s)-\tilde{u}_{n}(s)\right\|_{V} \\
\times\left\|u(s)-\tilde{u}_{n}(s)\right\| d s \\
\leq E \int_{0}^{\tau_{M}} y(s)\left(\|u(s)\|_{V}+\left\|\tilde{u}_{n}(s)\right\|_{V}\right)\|u(s)\|^{2}\left\|u(s)-\tilde{u}_{n}(s)\right\|_{V} d s \\
\leq \sqrt{2} M\left(\int_{0}^{\tau}\left(\|u(s)\|_{V}^{2}+\left\|\tilde{u}_{n}(s)\right\|_{V}^{2}\right) d s\right)^{\frac{1}{2}}\left(\int_{0}^{\tau} M u(s)-\tilde{u}_{n}(s) \|_{V}^{2} d s\right)^{\frac{1}{2}}
\end{gathered}
$$

and hence

$$
\lim _{n \rightarrow \infty} E \int_{0}^{\tau} y(s)\left\|\mathfrak{B}(u(s), u(s))-\mathfrak{B}\left(\tilde{u}_{n}(s), \tilde{u}_{n}(s)\right)\right\|_{V^{*}}^{2} d s=0
$$


For the subsequence $\left(n^{\prime}\right)$ of $(n)$ we have proved that $u_{n^{\prime}} \rightarrow u$ in $\mathcal{L}_{V}^{2}(\Omega \times[0, T])$. But $I_{\left[0, \tau_{M}\right]} \mathscr{B}(u, u), \mathscr{B}^{*} \in \mathcal{L}_{V^{*}}^{2}(\Omega \times[0, T])$, where $I_{\left[0, \tau_{M}\right]}$ denotes the indicator function of the interval $\left[0, \tau_{M}\right]$. Consequently,

$$
\begin{gathered}
\lim _{n^{\prime} \rightarrow \infty} E \int_{0}^{\tau} y(s)\left\langle\mathfrak{B}^{*}(s)-\mathscr{B}\left(\widetilde{u}_{n^{\prime}}(s), \tilde{u}_{n^{\prime}}(s), \widetilde{u}_{n^{\prime}}(s)-u_{n^{\prime}}(s)\right\rangle d s\right. \\
\lim _{n^{\prime} \rightarrow \infty} E \int_{0}^{\tau} M(s)\left\langle\mathfrak{B}^{*}(s)-\mathfrak{B}(u(s), u(s)), \widetilde{u}_{n^{\prime}}(s)-u_{n^{\prime}}(s)\right\rangle d s \\
+\lim _{n^{\prime} \rightarrow \infty} E \int_{0}^{\tau} y(s)\left\langle\mathscr{B}(u(s), u(s))-\mathfrak{B}\left(\widetilde{u}_{n^{\prime}}(s), \tilde{u}_{n^{\prime}}(s)\right), \tilde{u}_{n^{\prime}}(s)-u_{n^{\prime}}(s)\right\rangle d s=0 .
\end{gathered}
$$

Since $\mathrm{C} u_{n^{\prime}} \rightarrow \mathrm{C}^{*}$ in $\mathcal{L}_{V}^{2}(\Omega \times[0, T])$ and $\sum_{i=1}^{\infty}\left(\mathrm{C}^{*}(s)-\mathrm{C} u(s), h_{i}\right) h_{i}$ is the Fourier expansion of $\mathrm{C}^{*}(s)-\mathrm{C} u(s)$, the following relations hold:

$$
\begin{gathered}
\lim _{n^{\prime} \rightarrow \infty} E \int_{0}^{\tau}\left(\mathrm{C}^{*}(s)-\mathrm{C} u_{n^{\prime}}(s), \mathrm{C}^{*}(s)-\mathrm{C} u(s)\right)_{H_{n^{\prime}}} d s \\
=\lim _{n^{\prime} \rightarrow \infty} E \int_{0}^{\tau}\left(\mathrm{C}^{*}(s)-\mathrm{C} u_{n^{\prime}}(s), \sum_{i=1}^{n^{\prime}}\left(\mathrm{C}^{*}(s)-\mathrm{C} u(s), h_{i}\right) h_{i}\right) d s=0
\end{gathered}
$$

and

$$
\lim _{n^{\prime} \rightarrow \infty} E \int_{0}^{\tau_{M}}\left\|\mathrm{Cu}(s)-\mathrm{C}^{*}(s)\right\|_{H_{n^{\prime}}}^{2} d s=E \int_{0}^{\tau_{M}}\left\|\mathrm{Cu}(s)-\mathrm{C}^{*}(s)\right\|^{2} d s
$$

In view of these results, we see that by taking $n^{\prime} \rightarrow \infty$ in (21) the right-hand side of this inequality tends to zero. Therefore,

$\lim _{n^{\prime} \rightarrow \infty} E y\left(\tau_{M}\right)\left\|\tilde{u}_{n^{\prime}}\left(\tau_{M}\right)-u_{n^{\prime}}\left(\tau_{M}\right)\right\|^{2}=0, \lim _{n^{\prime} \rightarrow \infty} E \int_{0}^{\tau_{M}} y(s)\left\|\widetilde{u}_{n^{\prime}}(s)-u_{n^{\prime}}(s)\right\|_{V}^{2} d s=0$, and

$$
E \int_{0}^{\tau_{M}} y(s)\left\|\mathrm{e} u(s)-\mathrm{e}^{*}(s)\right\|^{2} d s=0
$$

From the properties of $y$ over $\left[0, \tau_{M}\right]$ and from (17) it thus follows that for each fixed natural number $M$,

$$
E \int_{0}^{\tau}\left\|u(s)-u_{n^{\prime}}(s)\right\|_{V}^{2} d s \rightarrow 0 \text { and } E\left\|u\left(\tau_{M}\right)-u_{n^{\prime}}\left(\tau_{M}\right)\right\|^{2} \rightarrow 0
$$


for $n^{\prime} \rightarrow \infty$.

Lemma 3.4: (i) The process $(u(t))_{t \in[0, T]}$ is a strong solution of the stochastic Navier-Stokes equations (2) and it has almost surely continuous trajectories in $H$.

(ii) The process $(u(t))_{t \in[0, T]}$ is with probability one a unique solution of equation (2).

Proof: (i) From (24), proved in Lemma 3.3., we conclude that

$$
I_{\left[0, \tau_{M}\right]}(s) \mathcal{C} u(s)=I_{\left[0, \tau_{M}\right]}(s) \mathcal{C}^{*}(s) \text { for a.e. }(\omega, t) \in \Omega \times[0, t] .
$$

The set

$$
\begin{gathered}
\text { D: }=\left\{x \in \mathcal{L}_{V}^{2}(\Omega \times[0, T]) \mid x=\beta v, v \in V ;\right. \\
\left.\beta \in \mathcal{L}_{\mathbb{R}}^{2}(\Omega \times[0, T]) \text { is for a.e. }(\omega, t) \text { bounded }\right\}
\end{gathered}
$$

is dense in $\mathcal{L}_{V}^{2}(\Omega \times[0, T])$. Using $(25)$ and the properties of $\mathscr{B}$ it can be proved that

$$
\lim _{n^{\prime} \rightarrow \infty} E \int_{0}^{\tau}\left\langle\mathscr{B}(u(s), u(s))-\mathscr{B}\left(u_{n^{\prime}}(s), u_{n^{\prime}}(s)\right), x\right\rangle d s=0 \text { for all } x \in \mathscr{D} \text {. }
$$

But $\mathscr{B}\left(u_{n^{\prime}}, u_{n^{\prime}}\right) \rightarrow \mathscr{B}^{*}$ in $\mathcal{L}_{V^{*}}^{2}(\Omega \times[0, T]) ;$ so,

$$
\lim _{n^{\prime} \rightarrow \infty} E \int_{0}^{\tau}\left\langle\mathscr{B}^{*}(s)-\mathscr{B}\left(u_{n^{\prime}}(s), u_{n^{\prime}}(s)\right), x\right\rangle d s=0 \text { for all } x \in \mathscr{D} .
$$

Since $\mathscr{D}$ is dense in $\mathscr{L}_{V}^{2}(\Omega \times[0, T])$, it follows that

$$
I_{\left[0, \tau_{M}\right]}(s) \mathscr{B}^{*}(s)=I_{\left[0, \tau_{M}\right]}(s) \mathscr{B}(u(s), u(s)) \text { for a.e. }(\omega, t) \in \Omega \times[0, T] .
$$

Using (26) and (27) in (3) we have that

$$
\begin{aligned}
\left(u\left(t \wedge \tau_{M}\right), v\right)+ & \int_{0}^{t \wedge \tau_{M}}\langle\mathcal{A} u(s), v\rangle d s=\left(x_{0}, v\right)+\int_{0}^{t \wedge \tau_{M}}\langle\mathscr{B}(u(s), u(s), v\rangle d s \\
& +\int_{0}^{t \wedge \tau_{M}}(f(s), v) d s+\int_{0}^{t \wedge \tau_{M}}(\mathrm{C} u(s), v) d w(s)
\end{aligned}
$$

for all $v \in V, t \in[0, T]$ and a.e. $\omega \in \Omega$.

From the properties of the stopping time $\tau_{M}$ and Lemma 3.2 we see that Let

$$
P\left(\bigcup_{M=1}^{\infty}\left\{\tau_{M}=T\right\}\right)=1-P\left(\bigcap_{M=1}^{\infty}\left\{\tau_{M}<T\right\}\right)=1-\lim _{M \rightarrow \infty} P\left(\tau_{M}<T\right)=1
$$




$$
\Omega^{\prime}:=\left\{\omega \in \Omega: \omega \in \bigcup_{M=1}^{\infty}\left\{\tau_{M}=T\right\} \text { and } u(\omega, t) \text { satisfies (28) for all } v \in V, t \in[0, T]\right\}
$$

Obviously, $P\left(\Omega^{\prime}\right)=1$.

Let $\omega \in \Omega^{\prime}$ be arbitrary and fixed. For this $\omega$, there exists a natural number $M_{0}$ such that $\tau_{M}=T$ for all $M>M_{0}$. From (28) we have

$$
\begin{aligned}
(u(t), v)+ & \int_{0}^{t}\langle\mathcal{A} u(s), v\rangle d s=\left(x_{0}, v\right)+\int_{0}^{t}\langle\mathscr{B}(u(s), u(s)), v\rangle d s \\
& +\int_{0}^{t}(f(s), v) d s+\int_{0}^{t}(\mathcal{C} u(s), v) d w(s)
\end{aligned}
$$

for all $v \in V, t \in[0, T]$. Consequently, (29) holds for all $\omega \in \Omega^{\prime}$. This means that the process $(u(t))_{t \in[0, T]}$ satisfies the Navier-Stokes equation (2). From Lemma 3.1, it follows that $u$ has almost surely continuous trajectories in $H$ and from Lemma 3.5 it follows that $E \sup _{t \in[0, T]}\|u(s)\|^{2}<\infty$. Hence $(u(t))_{t \in[0, T]}$ is a strong solution of the Navier-Stokes equation (2).

(ii) In order to prove the uniqueness we assume that $u, z \in \mathcal{L}_{V}^{2}(\Omega \times[0, T])$ are two solutions of equation (2), which have in $H$ almost surely continuous trajectories. Let

$$
y(t)=\exp \left\{-\frac{b}{\nu} \int_{0}^{t}\|u(s)\|_{V}^{2} d s-\lambda t\right\}
$$

for all $t \in[0, T]$ and a.e. $\omega \in \Omega$. It follows by the Itô formula that

$$
\begin{gathered}
y(t)\|u(t)-z(t)\|^{2}+2 \int_{0}^{t} y(s)\langle\mathcal{A} u(s)-\mathcal{A} z(s), u(s)-z(s)\rangle d s \\
\left.=2 \int_{0}^{t} y(s)\langle\mathfrak{B}(u(s), u(s))-\mathfrak{B}(z)(s), z(s)), u(s)-z(s)\right\rangle d s \\
-\frac{b}{\nu} \int_{0}^{t} y(s)\|u(s)\|_{V}^{2}\|u(s)-z(s)\|^{2} d s-\lambda \int_{0}^{t} y(s)\|u(s)-z(s)\|^{2} d s \\
+2 \int_{0}^{t} y(s)(\mathrm{C} u(s)-\mathrm{C} z(s), u(s)-z(s)) d w(s)+\int_{0}^{t} y(s)\|\mathrm{C} u(s)-\mathrm{C} z(s)\|^{2} d s .
\end{gathered}
$$

In view of the properties of $\mathscr{B}$ we can write

$$
2\langle\mathfrak{B}(u(s), u(s))-\mathscr{B}(z(s), z(s)), u(s)-z(s)\rangle=2\langle\mathscr{B}(u(s)-z(s), u(s)), u(s)-z(s)\rangle
$$




$$
\leq \frac{b}{\nu}\|u(s)\|_{V}^{2}\|u(s)-z(s)\|^{2}+\nu\|u(s)-z(s)\|_{V}^{2}
$$

Now we use the properties of $\mathcal{A}$ and $\mathcal{C}$ to obtain

$$
\begin{gathered}
y(t)\|u(t)-z(t)\|^{2}+\nu \int_{0}^{t} y(s)\|u(s)-z(s)\|_{V}^{2} d s \\
\leq 2 \int_{0}^{t} y(s)(\mathrm{C} u(s)-\mathrm{C} z(s), u(s)-z(s)) d w(s)
\end{gathered}
$$

for all $t \in[0, T]$ and a.e. $\omega \in \Omega$. Consequently,

$$
E y(t)\|u(t)-z(t)\|^{2}=0 \text { for all } t \in[0, T]
$$

and hence $P(u(t)=z(t))=1$ for all $t \in[0, T]$. Then for each countable and dense subset $\sigma \subset[0, T]$ we have

$$
P\left(\sup _{t \in \mathcal{T}}\|u(t)-z(t)\|=0\right)=1
$$

But $u$ and $z$ have almost surely continuous trajectories in $H$, so

$$
P\left(\sup _{t \in[0, T]}\|u(t)-z(t)\|=0\right)=1
$$

This means that (2) has an almost surely unique solution.

Lemma 3.5: There exists a positive constant $c_{2}$ (depending only on $\lambda, \nu$ and $T$ ) such that both expressions

$$
\sup _{t \in[0, T]} E\|u(t)\|^{4}, \quad E\left(\int_{0}^{T}\|u(s)\|_{V}^{2} d s\right)^{2}
$$

are less than or equal to $c_{2}\left[E\left\|x_{0}\right\|^{4}+E \int_{0}^{T}\|f(s)\|^{4} d s\right]$.

The proof of Lemma 3.5 is analogous to that of Lemma 3.1 ; so it will be omitted.

Now we can prove Theorem 2.3.

Let $\delta, \varepsilon>0$ be arbitrary and fixed. In Lemma 3.3 we have proved that for each fixed natural number $M$,

$$
E \int_{0}^{\tau_{M}}\left\|u(s)-u_{n^{\prime}}(s)\right\|_{V}^{2} d s \rightarrow 0, E\left\|u\left(\tau_{M}\right)-u_{n^{\prime}}\left(\tau_{M}\right)\right\|^{2} \rightarrow 0 \text { for } n^{\prime} \rightarrow \infty .
$$

From Lemma 3.2 we have

$$
\lim _{M \rightarrow \infty} P\left(\tau_{M}<T\right)=0 .
$$

Hence there exists a natural number $M_{0}$ such that

$$
P\left(\tau_{M_{0}}<T\right) \leq \frac{\varepsilon}{2}
$$


From Lemma 3.3 it follows for this $M_{0}$ that we have

$$
E \int_{0}^{\tau_{M_{0}}}\left\|u(s)-u_{n^{\prime}}(s)\right\|_{V}^{2} d s \rightarrow 0, E\left\|u\left(\tau_{M_{0}}\right)-u_{n^{\prime}}\left(\tau_{M_{0}}\right)\right\|^{2} \rightarrow 0 \text { for } n^{\prime} \rightarrow \infty .
$$

Consequently, there exists a natural number $n_{0}^{\prime}$ (depending on $\delta, \varepsilon, M_{0}, T$ ) such that

$$
\frac{1}{\delta} E \int_{0}^{\tau_{M_{0}}}\left\|u(s)-u_{n^{\prime}}(s)\right\|_{V^{2}}^{2} d s \leq \frac{\varepsilon}{2}, \frac{1}{\delta} E\left\|u\left(\tau_{M_{0}}\right)-u_{n^{\prime}}\left(\tau_{M_{0}}\right)\right\|^{2} \leq \frac{\varepsilon}{2}
$$

for all $n^{\prime} \geq n_{0}^{\prime}$. We can write

$$
\begin{aligned}
& P\left(\int_{0}^{T}\left\|u(s)-u_{n^{\prime}}(s)\right\|_{V}^{2} d s \geq \delta\right) \leq P\left(\tau_{M_{0}}<T\right) \\
& +P\left(\left\{\tau_{M_{0}}=T\right\} \wedge\left\{\int_{0}^{T}\left\|u(s)-u_{n^{\prime}}(s)\right\|_{V}^{2} d s \geq \delta\right\}\right) \\
& \quad \leq \frac{\varepsilon}{2}+P\left(\int_{0}^{\tau_{M_{0}}}\left\|u(s)-u_{n^{\prime}}(s)\right\|_{V}^{2} d s \geq \delta\right) \\
& \leq \frac{\varepsilon}{2}+\frac{1}{\delta} E \int_{0}^{\tau}\left\|u(s)-u_{M^{\prime}}(s)\right\|_{V}^{2} d s<\frac{\varepsilon}{2}+\frac{\varepsilon}{2}=\varepsilon
\end{aligned}
$$

and

$$
\begin{gathered}
P\left(\left\|u(T)-u_{n^{\prime}}(T)\right\|^{2} \geq \delta\right) \leq P\left(\tau_{M_{0}}<T\right) \\
+P\left(\left\{\tau_{M_{0}}=T\right\} \wedge\left\{\left\|u(T)-u_{n^{\prime}}(T)\right\|^{2} \geq \delta\right\}\right) \\
\leq \frac{\varepsilon}{2}+P\left(\left\|u\left(\tau_{M_{0}}\right)-u_{n^{\prime}}\left(\tau_{M_{0}}\right)\right\|^{2} \geq \delta\right) \\
\leq \frac{\varepsilon}{2}+\frac{1}{\delta} E\left\|u\left(\tau_{M_{0}}\right)-u_{n^{\prime}}\left(\tau_{M_{0}}\right)\right\|^{2}<\frac{\varepsilon}{2}+\frac{\varepsilon}{2}=\varepsilon
\end{gathered}
$$

for all $n^{\prime} \geq n_{0}^{\prime}$. Hence for all $\delta>0$,

$$
P\left(\int_{0}^{T}\left\|u(s)-u_{n^{\prime}}(s)\right\|_{V}^{2} d s \geq \delta\right) \rightarrow 0, P\left(\left\|u(T)-u_{n^{\prime}}(T)\right\|^{2} \geq \delta\right) \rightarrow 0
$$


Therefore, the sequences $\left(\int_{0}^{T}\left\|u(s)-u_{n^{\prime}}(s)\right\|_{V}^{2} d s\right)$ and $\left(\left\|u(T)-u_{n^{\prime}}(T)\right\|^{2}\right)$ converge in probability to zero. From Lemma 3.1 and Lemma 3.5, it follows that these sequences are uniformly integrable (with respect to $\omega \in \Omega$ ). Hence,

$$
E \int_{0}^{T}\left\|u(s)-u_{n^{\prime}}(s)\right\|_{V}^{2} d s \rightarrow 0, \quad E\left\|u(T)-u_{n^{\prime}}(T)\right\|^{2} \text { for } n^{\prime} \rightarrow \infty .
$$

Every subsequence of $\left(u_{n}\right)$ has a further subsequence, which converges in the norm of the space $\mathcal{L}_{V}^{2}(\Omega \times[0, T])$ to the same limit $u$, the unique solution of the NavierStokes equation (2) (because we can repeat all arguments of the results of Section 3 for this subsequence). Applying a property from functional analysis (see [18, Proposition 10.13 , p. 480$)$, it follows that the whole sequence $\left(u_{n}\right)$ converges in mean square to $u$, i.e.,

$$
E \int_{0}^{T}\left\|u(s)-u_{n}(s)\right\|_{V}^{2} d s \rightarrow 0 \text { for } n \rightarrow \infty
$$

By the same argument we can prove that the whole sequence $\left(u_{n}(T)\right)$ converges to $u(T)$ in the norm of the space $\ell_{H}^{2}(\Omega)$, as soon as for all $t \in[0, T],\left(u_{n}(t)\right)$ converges to $u(t)$ in the norm of the space $\mathcal{L}_{H}^{2}(\Omega)$.

\section{Appendix - Some Convergence Principles}

For the convenience of the reader, we recall some basic convergence results.

Proposition 4.1: [19, Proposition 21.27, p. 261] Let $S_{1}$ and $S_{2}$ be Banach spaces and let $L: S_{1} \rightarrow S_{2}$ be a continuous linear operator. If $\left(x_{n}\right)$ is a sequence in $S_{1}$ such that $x_{n} \rightarrow x$ (where $\left.x \in S_{1}\right)$, then $L\left(x_{n}\right) \rightarrow L(x)$.

By applying Proposition 4.1, we obtain the following corollary.

Corollary 4.2: If $S$ is a Banach space and if $\left(x_{n}\right)$ is a sequence from $\mathcal{L}_{S}^{2}(\Omega \times[0, T])$, which converges weakly to $x \in \mathcal{L}_{S}^{2}(\Omega \times[0, T])$, then for $n \rightarrow \infty$ the following assertions are true:

(i)

$$
\int_{0}^{t} x_{n}(s) d s \rightarrow \int_{0}^{t} x(s) d s \text { and } \int_{0}^{t} x_{n}(s) d w(s) \rightarrow \int_{0}^{t} x(s) d w(s) \text { in } \mathcal{L}_{S}^{2}(\Omega \times[0, T])
$$

$$
\int_{0}^{T} x_{n}(s) d s \rightarrow \int_{0}^{T} x(s) d s \text { and } \int_{0}^{T} x_{n}(s) d w(s) \rightarrow \int_{0}^{T} x(s) d w(s) \text { in } \ell_{S}^{2}(\Omega) .
$$




\section{References}

[1] Bensoussan, A., Stochastic Navier-Stokes equations, Acta Appl. Math. 38:3 (1995), 267-304.

[2] Capinski, M. and Cutland, N.J., Nonstandard Methods for Stochastic Fluid Mechanics, World Scientific Publishing Co. 1995.

[3] Capinski, M. and Gatarek, D., Stochastic equations in Hilbert space with application to Navier-Stokes equations in any dimension, J. Funct. Anal. 126:1 (1994), 26-35.

[4] Flandoli, F. and Tortorelli, V.M., Time discretization of Ornstein-Uhlenbeck equations and stochastic Navier-Stokes equations with a generalized noise, Stoch. and Stoch. Reports 55:1-2 (1995), 141-165.

[5] Grecksch, W. and Kloeden, P.E., Time-discretized Galerkin approximations of stochastic parabolic PDEs, Bull. Austral. Math. Soc. 54 (1996), 79-85.

[6] Grecksch, W. and Schmalfuß, B., Approximation of the stochastic Navier-Stokes equation, Compl. Appl. Math. 15:3 (1997), 227-239.

[7] Grecksch, W. and Tudor, C., Stochastic Evolution Equations, Akademie Verlag, Berlin 1995.

[8] Gyöngy, I., On the approximation of stochastic partial differential equations I, II., Stochastics 25:2 (1988), 53-85, Stochastics 26:3 (1989), 129-164.

[9] Karatzas, I. and Shreve, S.E., Brownian Motion and Stochastic Calculus, Springer Verlag, New York, Berlin 1996.

[10] Komech, A.I. and Vishik, M.I., Statistical solutions of the Navier-Stokes and Euler equations, Adv. in Mechanics 5:1-2 (1982), 65-120. (Russian)

[11] Krylov, N.V. and Rozovskij, B.L., Ob evoljucionnych stochastičeskich uravnenijach. In: Itogi nauki i techniki (Glavn. Red. R.V. Gamkrelidze), Serija sovremennych problemy matematiki 14, VINITI, Moskva 1979. (Russian).

[12] Lions, J.L., Optimal Control of Systems Governed by Partial Differential Equations, Springer Verlag, Berlin, New York 1971.

[13] Pardoux, E., Equations aux derivées partielles stochastiques non linéaires monotones. Etude de solutions fortes de type Itô, Thése doct. Sci. Math. Univ. Paris 1975.

[14] Schmalfuß, B., Endlichdimensionale Approximation der stochastischen NavierStokes-Gleichung, Statistics 2 (1990), 149-157.

[15] Schmalfuß, B., Das Langzeitverhalten der stochastischen Navier-StokesGleichung, Habilitationsschrift, Technische Hochschule Merseburg 1992.

[16] Temam, R., Navier-Stokes Equations: Theory and Numerical Analysis, NorthHolland, Amsterdam 1979.

[17] Viot, M., Solution faible d'équation aux derivées partielles stochastiques non linéares, Thése doct. Sci. Math. Paris 1976.

[18] Zeidler, E., Nonlinear Functional Analysis and its Applications Vol. I: FixedPoint Theorems, Springer-Verlag, Berlin, New York 1986.

[19] Zeidler, E., Nonlinear Functional Analysis and its Applications Vol. II/A, II/B: Linear Monotone Operators, Springer-Verlag, Berlin, New York 1990. 


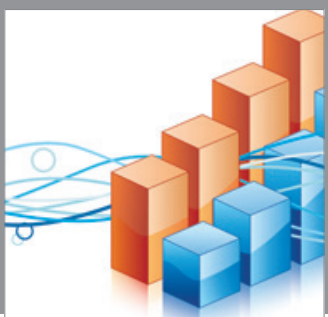

Advances in

Operations Research

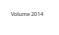

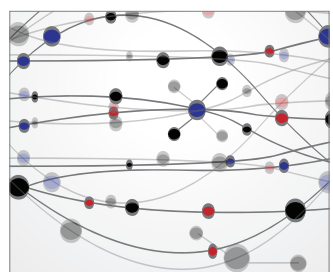

\section{The Scientific} World Journal
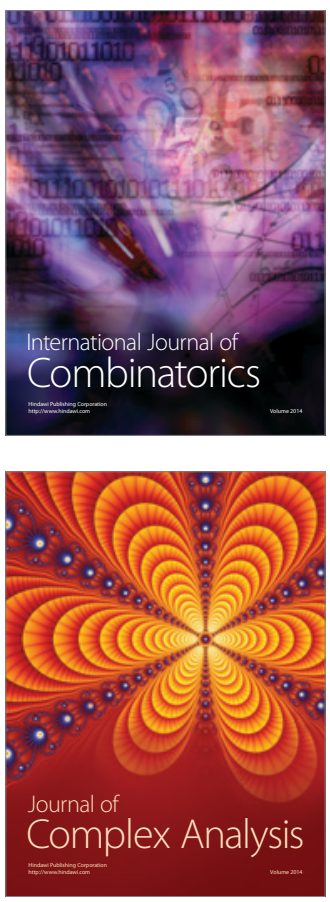

International Journal of

Mathematics and

Mathematical

Sciences
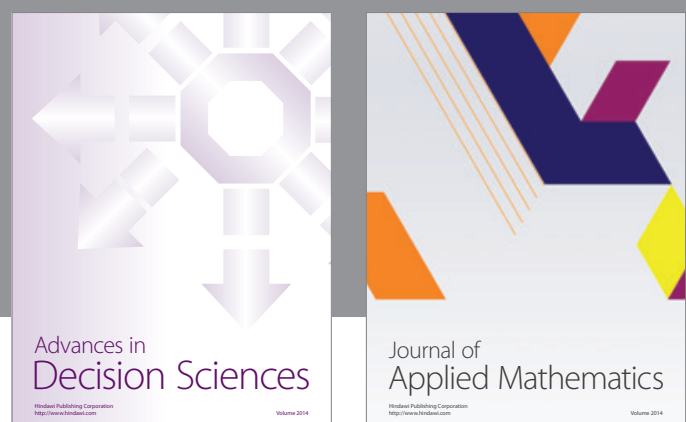

Journal of

Applied Mathematics
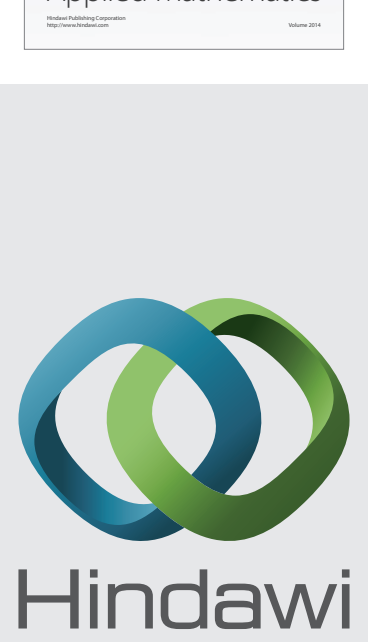

Submit your manuscripts at http://www.hindawi.com
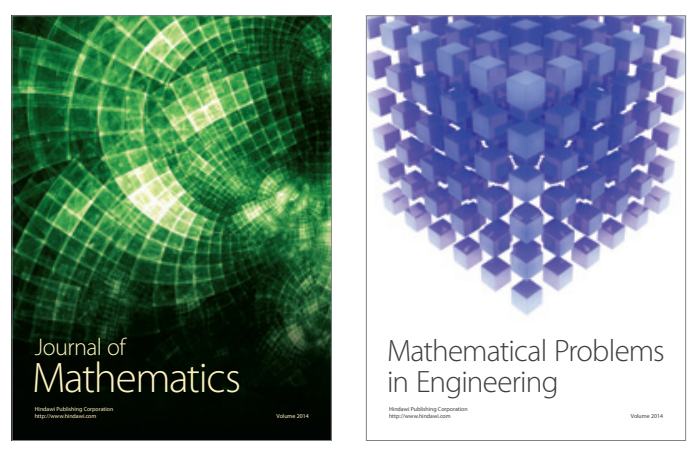

Mathematical Problems in Engineering
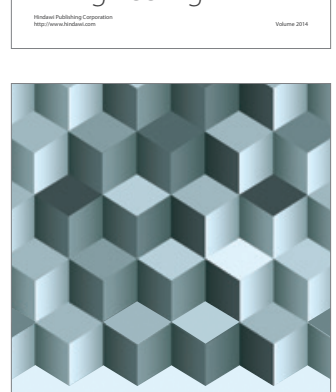

Journal of

Function Spaces
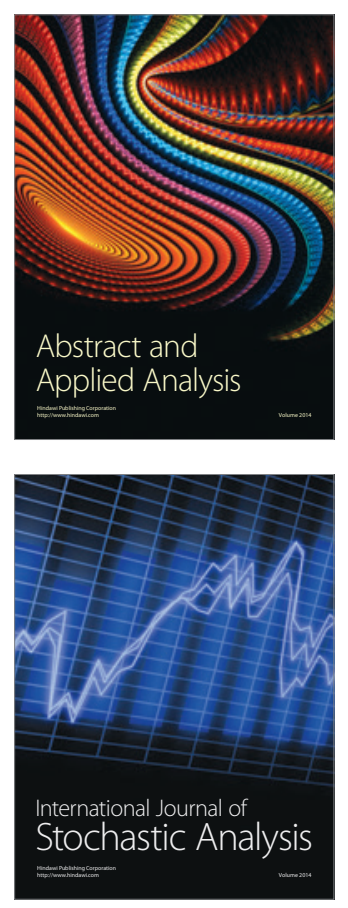

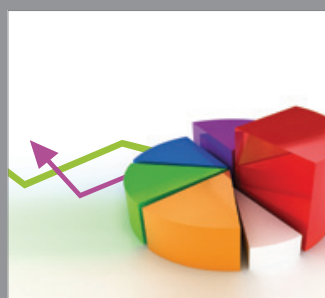

ournal of

Probability and Statistics

Promensencen
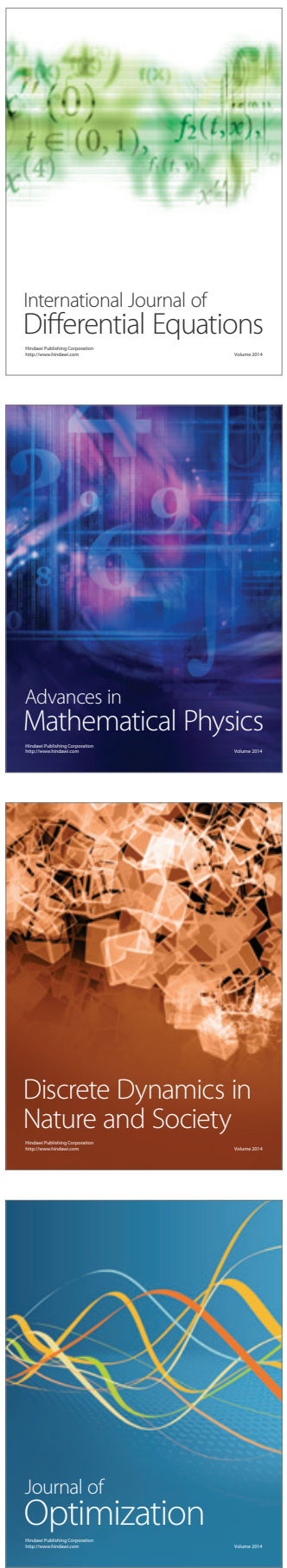